\title{
Monitoramento da taxa de saturação de oxigênio no sangue e frequência cardíaca via método de magnificação de vídeo Euleriana sem contato físico.
}

\section{Monitoring of blood oxygen saturation rate and heart rate via Eulerian video magnification method noncontact.}

\author{
Alessandra de Fátima Galvão Rosa ${ }^{\mathbb{D}, 1,2}$ and Roberto Cesar Betini ${ }^{(\mathbb{D}, 1}$ \\ ${ }^{1}$ Universidade Tecnológica Federal do Paraná - UTFPR, ${ }^{2}$ Instituto Carlos Chagas - ICC/FIOCRUZ \\ *alessandrarosa@alunos.utfpr.edu.br; betini@utfpr.edu.br
}

Recebido: 02/03/2019. Revisado: 20/04/2020. Aceito: 22/06/2020.

\section{Resumo}

$\mathrm{O}$ monitoramento da frequência cardíaca $(\mathrm{FC})$ e da taxa de saturação de oxigênio no sangue $\left(\mathrm{SpO}_{2}\right)$ é fundamental em cirurgias e unidades de terapia intensiva. Nas últimas décadas surgiram vários estudos sobre monitoramento de sinais vitais sem contato físico, mas poucos são focados na $\mathrm{SpO}_{2}$. Este artigo apresenta uma solução para o monitoramento da taxa de saturação de oxigênio no sangue e frequência cardíaca sem contato físico com o paciente através da técnica de fotopletismografia por imagens. A magnificação de vídeo Euleriana (EVM) foi empregada na solução proposta para ampliar as variações de cores que ocorrem na pele devido ao ciclo circadiano. O software desenvolvido foi executado em uma plataforma de hardware livre e de baixo custo. Foram realizados experimentos com nove indivíduos saudáveis. Ao comparar os resultados experimentais com os obtidos com um oxímetro de pulso de referência, a solução proposta mostrou boa acurácia nas medidas de $\mathrm{SpO}_{2}$, com diferença menor que $2 \%$, atendendo os requisitos da norma que regulamenta os equipamentos de oximetria de pulso.

Palavras-Chave: Frequência cardíaca, iPPG, monitoramento sem contato, saturação periférica de oxigênio, $\mathrm{SpO}_{2}$.

\section{Abstract}

Heart rate and peripheral oxygen saturation $\left(\mathrm{SpO}_{2}\right)$ monitoring is critical during surgeries and in intensive care units. Over the last decades, several studies have addressed noncontact monitoring of vital signs, but few reports have focused on $\mathrm{SpO}_{2}$. This article presents an Heart rate and $\mathrm{SpO}_{2}$ monitoring method without physical contact with the patient using imaging photoplethysmography. Eulerian video magnification (EVM) was employed in the proposed solution to amplify the changes in skin color due to the heart cycle. The developed software operates on a widely available, low-cost open hardware platform. Experiments were performed with nine healthy individuals. When comparing the experimental results to those obtained with a reference pulse oximeter, the proposed solution showed good accuracy in $\mathrm{SpO}_{2}$ measurements, with a difference of less than $2 \%$, meeting the requirements of the standard that regulates pulse oximetry equipment.

Keywords: Heart rate, iPPG, noncontact monitoring, peripheral oxygen saturation, $\mathrm{SpO}_{2}$. 


\section{Introdução}

A taxa de saturação de oxigênio $\left(\mathrm{SpO}_{2}\right)$ e a frequência cardíaca (FC) são dois parâmetros vitais para definir o status cardiovascular humano e o estado de saúde do paciente (Fan and Li, 2018, O'Driscoll et al., 2008).

Uma oxigenação adequada dos tecidos humanos é necessária para a manutenção da vida. Portanto, seu monitoramento contínuo é fundamental em cirurgias ou em internações em unidades de terapia intensiva UTI (Nitzan and Taitelbaum, 2008). A saturação de oxigênio é a quantidade de hemoglobina oxigenada do total de hemoglobina presente em $100 \mathrm{ml}$ de sangue. A hemoglobina é uma proteína responsável por levar o oxigênio dos pulmões até as células, pois, sem o suprimento adequado de oxigênio às células, os tecidos do corpo morrem (Edwards Life Sciences, 2002).

Segundo Guyton and John (2010) o trabalho sistólico do coração (stroke work output) é a quantidade de energia que o coração converte em trabalho a cada batimento, ao bombear o sangue para as artérias. 0 trabalho sistólicominuto (minute work output) é a quantidade total de energia convertida em trabalho em 1 minuto, ou seja, o resultado do trabalho produzido multiplicado pelo número de batimentos por minuto (bpm) também conhecido como frequência cardíaca (FC). Alguns dos tipos mais preocupantes de mau funcionamento cardíaco ocorrem devido ao ritmo cardíaco anormal, conhecido como arritmias. O monitoramento da FC indica o estado do ritmo cardíaco do paciente.

Para esse artigo o trabalho de Simioni (2015) referente a obtenção da FC sem contato físico foi adaptado e implementado em uma plataforma de hardware mais atualizada. Para a avaliação da acurácia do monitoramento da FC pela solução proposta o experimento descrito por Simioni (2015) foi reproduzido e as mesmas métricas foram utilizadas. Também foi verificada a concordância entre a solução proposta e um oxímetro de pulso comercial. Para tal, os valores da FC apresentados por ambos os métodos foram comparados através do método de concordância entre dois métodos de Bland-Altman apresentado por Altman and Bland (1983).

A capacidade de obter sinais fisiológicos remotamente do paciente possibilita reduzir custos, diminuir o fluxo de trabalho, reduzir a necessidade de cabeamento e equipamentos em contato com a pele do paciente e, portanto, traz mais liberdade para o paciente (Addison et al., 2017), também evita a contaminação cruzada entre pacientes e dos equipamentos em caso de doenças infectocontagiosas. Considerando a necessidade de monitoramento contínuo da $\mathrm{SpO}_{2}$ e da $\mathrm{FC}$, em condições que o contato físico deve ser evitado, como, por exemplo, em vítimas de queimaduras, esse artigo propõe uma solução de monitoramento da $\mathrm{SpO}_{2}$ e a FC sem contato físico.

Visando contribuir para a melhora da qualidade de vida dos indivíduos que necessitam de monitoramento contínuo, e na redução dos custos para o sistema de saúde ao evitar o surgimento de lesões decorrente do contato com os equipamentos de monitoramento, a solução proposta utiliza imagens de vídeo para a obtenção dos sinais vitais e foi desenvolvida em software livre e foi executada em uma plataforma de hardware livre e de baixo custo.

A fotopletismografia baseada em câmera de vídeo ou image Photoplethysmography (iPPG) é uma técnica que pode ser empregada para medir sinais vitais sem contato físico (Verkruysse et al., 2008, Tarassenko et al., 2014, Shao et al., 2016). Para verificar se o sistema proposto nesse artigo pode ser uma alternativa sem contato físico aos oxímetros de pulso com contato, o objetivo dessa pesquisa é avaliar a utilização de um sistema de monitoramento contínuo da $\mathrm{SpO}_{2}$ e da FC com as seguintes características:

- Operar sem contato físico;

- Obter e processar sinais fotopletismográficos;

- Estimar a taxa de saturação de oxigênio e frequência cardíaca online;

- Ser implementado em uma plataforma de hardware de baixo custo;

- Utilizar software livre e gratuito.

\section{Trabalhos relacionados}

Os estudos relacionados com a obtenção da $\mathrm{SpO}_{2}$ sem contato físico utilizando imagens de vídeo foram identificados através do processo de mapeamento sistemático, o qual retornou 791 artigos. Após a aplicação dos critérios de inclusão e exclusão, definidos no protocolo do mapeamento, o resultado obtido foi uma lista com 17 estudos correlatos. Posteriormente foram adicionados a lista de estudos relacionados os estudos de Moço et al. (2018) e Wang et al. (2018) após a análise das referência bibliográficas da revisão sistemática apresentada por Tamura (2019). Também foram adicionados os estudo de Poh and Poh (2017), Kooij and Naber (2019). Totalizando 21 estudos correlatos que são detalhados na Tabela 1.

O primeiro estudo sobre o tema é de Wieringa et al. (2005), porém os autores de Van Gastel et al. (2016) afirmam que o estudo de Wieringa et al. (2005) não apresentou os resultados da $\mathrm{SpO}_{2}$, sendo que o primeiro a apresentar os resultados foi Humphreys et al. (2007).

Os estudos mapeados foram divididos em duas categorias, os que utilizam e os que não utilizam fonte de luz específica. Do total final de estudos primários mapeados apenas Kong et al. (2013), Imms et al. (2014), Tarassenko et al. (2014), Bal (2015), Van Gastel et al. (2016), Poh and Poh (2017), Fan and Li (2018), Moço et al. (2018) e Wang et al. (2018) utilizam luz ambiente. O uso da luz ambiente torna a solução mais versátil, facilita sua replicação e possibilita a utilização em diversos ambientes.

De todos os estudos elencados apenas Humphreys et al. (2007), Tarassenko et al. (2014), Bal (2015), Addison et al. (2017), Cobos-Torres and Abderrahim (2017), Fan and Li (2018) e Moço et al. (2018) realizam o monitoramento da $\mathrm{SpO}_{2}$ e da FC.

Outra característica analisada nos estudos correlatos é a obtenção da $\mathrm{SpO}_{2}$ e FC em tempo real, ou seja, o valor da $\mathrm{SpO}_{2}$ e $\mathrm{FC}$ é aferido simultaneamente à gravação dos vídeos. O trabalho de Cobos-Torres and Abderrahim 
Tabela 1: Estudos correlatos sobre a mensuração da $\mathrm{SpO}_{2}$ e FC sem contato físico.

\begin{tabular}{|c|c|c|c|c|c|}
\hline Câmera & Fonte de luz ${ }^{1}$ & Simultâneo $^{2}$ & Part. 3 & Sinal aferido 4 & Estudo \\
\hline $1 \mathrm{CMOS}$ & $1 \mathrm{LI}$ & Não & $7^{*}$ & $\mathrm{SpO}_{2}$ & $\begin{array}{l}\text { Wieringa et al. } \\
(2005)\end{array}$ \\
\hline $1 \mathrm{CMOS}$ & 1 ALED & Não & $10^{*}$ & $\mathrm{SpO}_{2}$ e $\mathrm{FC}$ & $\begin{array}{l}\text { Humphreys et al. } \\
(2007)\end{array}$ \\
\hline 1 Color CCD & 2 ALED & Não & Não especificado & $\mathrm{SpO}_{2}$ & Wang et al. (2010) \\
\hline 2 Mono & LA & Não & $30^{*}$ & $\mathrm{SpO}_{2}$ & Kong et al. (2013) \\
\hline 1 Mono & 5 LED & Não & $5 *$ & $\mathrm{SpO}_{2}$ & Li et al. (2013) \\
\hline $1 \mathrm{CMOS}$ & $1 \mathrm{AL}$ & Não & Não especificado & $\mathrm{SpO}_{2}$ & Imms et al. (2014) \\
\hline 1 Color HD & LA & Não & $46^{*}$ & $\mathrm{SpO}_{2}, \mathrm{FC}$ e FR & $\begin{array}{l}\text { Tarassenko et al. } \\
(2014)\end{array}$ \\
\hline 1 Mono & $1 \mathrm{AL}$ & Não & $9 *$ & $\mathrm{SpO}_{2}$ & Tsai et al. (2014) \\
\hline 1 Webcam & LA & Não & $6 *$ e $3 * *$ & $\mathrm{SpO}_{2}$ e FC & Bal (2015) \\
\hline 1 Color CCD & $2 \mathrm{ML}$ & Não & $5^{*}$ & $\mathrm{SpO}_{2}$ & Guazzi et al. (2015) \\
\hline $1 \mathrm{CMOS}$ & 1 ALED & Não & $6^{*}$ & $\mathrm{SpO}_{2}$ & Shao et al. (2016) \\
\hline 3 Mono & LA & Não & $4^{*}$ & $\mathrm{SpO}_{2}$ & $\begin{array}{c}\text { Van Gastel et al. } \\
(2016)\end{array}$ \\
\hline 1 Color HD & $1 \mathrm{LC}$ & Não & $8 * * *$ & $\mathrm{SpO}_{2}, \mathrm{FC}$ e FR & Addison et al. (2017) \\
\hline $1 \mathrm{CMOS}$ & $1 \mathrm{ML}$ & Sim & $10^{*}$ & $\mathrm{SpO}_{2}$ e FC & $\begin{array}{c}\text { Cobos-Torres and } \\
\text { Abderrahim (2017) }\end{array}$ \\
\hline 1 Webcam color & $1 \mathrm{AL}$ & Não & $15^{*}$ & $\mathrm{SpO}_{2}$ & Mishra et al. (2017) \\
\hline 1 iPhone & LA & Sim & $40 *$ & $\mathrm{FC}$ & Poh and Poh (2017) \\
\hline 2 Mono & $18 \mathrm{LI}$ & Não & $51^{*}$ & $\mathrm{SpO}_{2}$ & $\begin{array}{l}\text { Verkruysse et al. } \\
\qquad(2017)\end{array}$ \\
\hline 1 Color CCD & LA & Não & $65^{*}$ & $\mathrm{SpO}_{2}$ e FC & Fan and $\mathrm{Li}(2018)$ \\
\hline 1 Mono e 1 CMOS & LA e LH & Não & $16^{*}$ & $\mathrm{SpO}_{2}$ e FC & Moço et al. (2018) \\
\hline $\begin{array}{l}1 \text { Color CCD e } \\
1 \text { Infravermelho }\end{array}$ & LA, 2 LI e LF & Não & $22 *$ e $1 * *$ & $\mathrm{FC}$ & Wang et al. (2018) \\
\hline 1 Webcam color & $1 \mathrm{CL}$ & Não & $21^{*}$ & FC & $\begin{array}{l}\text { Kooij and Naber } \\
\text { (2019) }\end{array}$ \\
\hline
\end{tabular}

${ }^{1}$ Fonte de luz: LI: lâmpada incandescente; ALED: array de LED; LA: luz ambiente; LED: diodo emissor de luz (Light Emitting Diode); AL: anel de LED; ML: mosaico de LED; CL: caixa de luz; LC: lâmpada cirúrgica; LH: lâmpada de halogêneo; LF: lâmpada fluorescente.

2 Simultâneo: o sinal fisiológico aferido é apresentado pela solução proposta simultaneamente à gravação do vídeo.

3 Tipo participante: *humano saudável; **Paciente em UTI pediátricas; ***porcos saudáveis.

4 Sinal vital aferido: $\mathrm{SpO}_{2}$ : saturação de oxigênio, $\mathrm{FC}$ : frequência cardíaca e FR: frequência respiratória.

(2017) é o único estudo que menciona que realiza $100 \%$ do processamento online. O processamento online ou em tempo real faz a solução sem contato físico se assemelhar aos oxímetros de pulso com contato físico. Essa característica pode facilitar a aceitação da solução como uma alternativa sem contato aos oxímetros de pulso convencionais. O trabalho de Poh and Poh (2017) apresenta o monitoramento através da face em tempo real, mas nesse estudo é utilizado um software proprietário de código fechado chamado "Cardiio Heart Rate app" desenvolvido apenas para a plataforma de hardware iPhone, tal característica limita o acesso código fonte da aplicação e a aplicação não obtém a $\mathrm{SpO}_{2}$.

Dos artigos obtidos desse mapeamento nenhum dos estudos utiliza a técnica de magnificação de vídeo Euleriana (EVM). Conforme afirma Verkruysse et al. (2017) poucos estudos são focados na obtenção da $\mathrm{SpO}_{2}$. O uso de imagens de vídeo para o processamento de sinais fotopletismográficos é recente portanto, novos estudos devem ser propostos para trazer avanços para as soluções não invasivas e sem contato físico que monitoram sinais vitais.

\section{Materiais e métodos}

As seções a seguir apresentam os materiais e métodos utilizados nesse artigo para a avaliação da solução proposta para a obtenção da $\mathrm{SpO}_{2}$ e da frequência cardíaca sem contato físico e de baixo custo.

\subsection{População}

Nove indivíduos saudáveis ( 5 pessoas do sexo feminino e 4 pessoas do sexo masculino), com idade entre: 23 e 54 anos (idade média: $33,7, \pm$ desvio-padrão: $\pm 10,0$ ), não fumantes, com tipo de pele entre: II e VI, participaram da pesquisa. Todos receberam orientações sobre a pesquisa e sobre os procedimentos realizados nos experimentos. Todos assinaram o termo de livre consentimento e responderam ao questionário de características físicas onde informaram idade, sexo, se eram fumantes e responderam questões relativas ao comportamento da pele quando exposta ao sol para a definição do tipo de pele, conforme a escala Fitzpatrick descrita em Fitzpatrick (1988). 


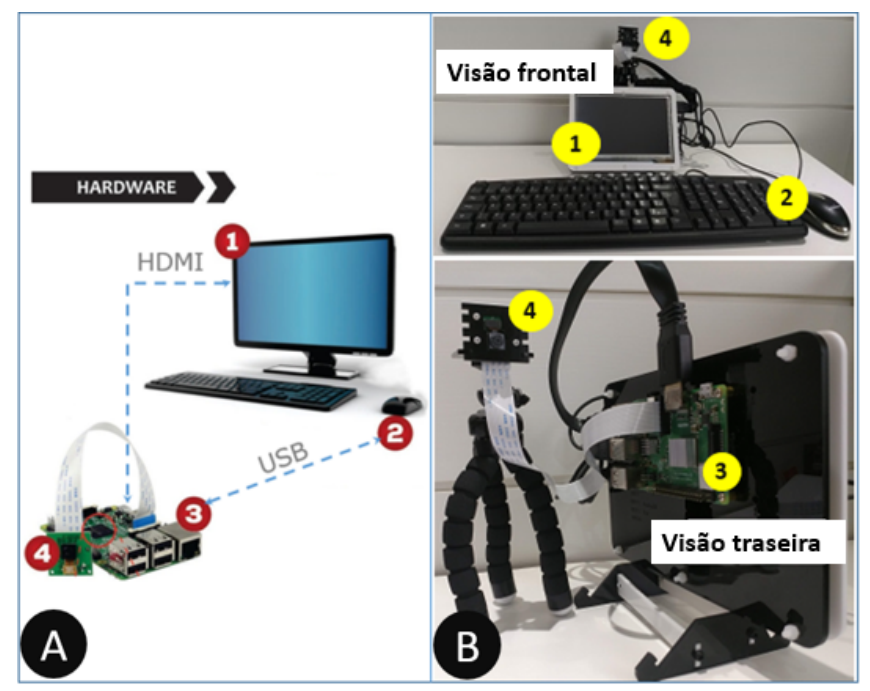

Figura 1: (A) Diagrama de hardware proposto. (B) Imagem real do protótipo.

\subsection{Hardware}

A Fig. 1 item (A) apresenta o diagrama de hardware da solução proposta, o item (B) ilustra a imagem real do protótipo da solução. O destaque (1) corresponde ao monitor HDMI, (2) mouse e teclado, (3) Raspberry Pi 3 e (4) Raspberry Pi Camera. Para a realização dos experimentos foi utilizada apenas a luz ambiente. Todos os vídeos foram capturados com uma taxa de captura de 30 fps (frame por segundo).

Durante os experimentos o indivíduo foi monitorado com o oxímetro de pulso (Geratherm modelo: GT300C203. $\mathrm{SpO}_{2}$ : Faixa de mensuração: 70-99\%; Acurácia: $\pm 2 \%$ entre $80 \%$ e $99 \% ; \pm 3 \%$ entre $70 \%$ e $80 \%$. FC: Intervalo de medição: 30 a 250 bpm; Precisão: 30 a 99 bpm, \pm 2 bpm; 100 a 250, \pm 2 bpm; Resolução: 1 bpm), o qual foi utilizado como método de referência para obtenção da $\mathrm{SpO}_{2}$ e FC.

\subsection{Desenho experimental}

Para a realização dos experimentos os indivíduos se posicionaram em frente a câmera a uma distância de aproximadamente sessenta centímetros. Foi solicitado que o oxímetro de pulso fosse colocado em um dos dedos de uma das mãos e que o posicionamento da mão ficasse de tal forma que a câmera da solução proposta pudesse capturar o display do oxímetro, e que fosse uma posição confortável para o indivíduo. A câmera foi conectada à um Raspberry Pi 3 Modelo $\mathrm{B}+$, onde o software da solução proposta foi executado como mostrado na Fig. 2, onde (B1) corresponde as leituras da $\mathrm{SpO}_{2}$ e (B2) as leituras da FC.

\subsection{Calibração}

Para obter a $\mathrm{SpO}_{2}$ é necessário realizar uma calibração antes do monitoramento de cada indivíduo. Sendo as-

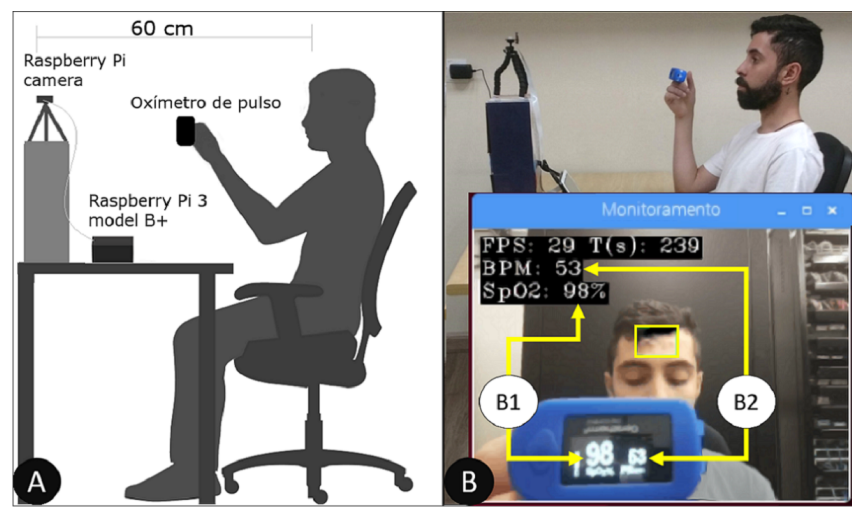

Figura 2: (A) Esquema dos experimentos. (B) Imagem real do esquema do experimento.

sim, quando o software da solução proposta é iniciado é apresentada a seguinte pergunta: "É calibração?", com as seguintes opções: (s/n/r). A opção "s" corresponde a "Sim, é calibração", "n" corresponde a "Não é calibração", neste caso o sistema realiza o monitoramento da $\mathrm{SpO}_{2}$, e " $\mathrm{r}$ " corresponde ao cálculo da regressão linear. O cálculo da regressão linear é necessário para definir os coeficientes alfa e beta utilizados na equação para obtenção da $\mathrm{SpO}_{2}$. Ao escolher a opção "s" é iniciado o procedimento de calibração. Para tal, o software solicita a identificação do voluntário. $O$ processo de calibração compreende na gravação de dois vídeos de dois minutos de duração cada. O software pergunta se é a gravação do primeiro ou do segundo vídeo conforme mostra a Fig. 3, ao solicitar a sequência de calibração.

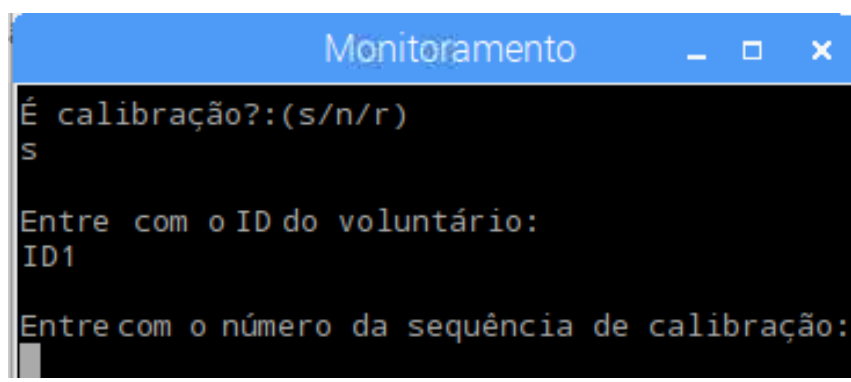

Figura 3: Tela inicial da calibração do software proposto.

Essa identificação do voluntário e da sequência do vídeo é necessária pois o software cria os arquivos: (RR_XXXX_1.txt e SpO2_XXXX_1.txt) para o primeiro vídeo, e ( $\mathrm{R} \overline{\mathrm{R}} \mathrm{XXXX} \_2 . \overline{\mathrm{x}} \mathrm{t}$ e $\left.\mathrm{Sp} \overline{\mathrm{O}} 2 \_\mathrm{XXXX} \_2 . t \mathrm{xt}\right)$ para o segundo vídeo. O campo XXXX no nome do arquivo corresponde a identificação do voluntário. Com a identificação correta de cada arquivo são evitados erros no armazenamento dos dados capturados durante a execução dos experimentos, e é uma forma de registrar as informações relativas aos experimentos para cada voluntário.

Após o início de cada procedimento de calibração 
a cada 10 segundos o valor da taxa $R$, obtida pelo software é armazenado no arquivo RR_XXXX_1.txt ou RR_XXXX 2.txt conforme sequência de calibração informada. No mesmo instante de tempo é armazenado manualmente o valor da $\mathrm{SpO} 2$ apresentado pelo oxímetro de referência no arquivo SpO2_XXXX_1.txt ou $\mathrm{SpO} 2$ XXXX_2.txt.

No primeiro vídeo de calibração foi solicitado que o participante respirasse normalmente durante os 120 segundos, que corresponde a duração total do procedimento. No segundo vídeo de calibração foi solicitado que após decorridos os primeiros 10 segundos do início do procedimento, o participante segurasse a respiração até se sentir desconfortável. Decorrido esse período que o voluntário ficou sem respirar (o qual variou de 10 a 30 segundos entre os voluntários) foi solicitado que o participante respirasse normalmente até o final dos 120 segundos do procedimento.

O processo de calibração foi efetuado uma vez para cada indivíduo. Finalizado o processo de calibração é necessário iniciar o software para realizar o cálculo dos coeficientes alfa e beta usados para o cálculo da $\mathrm{SpO}_{2}$, conforme Eq. (1).

\subsubsection{Coeficiente alfa e beta}

O cálculo da regressão linear tem como propósito definir os valores dos coeficientes $\alpha$ e $\beta$, que são utilizados na Eq. (1). Onde R é razão das taxas de absorbância da intensidade de cor dos comprimentos de onda de luz vermelha e azul obtidas no processo de calibração, $\alpha$ é a inclinação da reta de regressão estimada e $\beta$ é o ponto em que a reta de regressão estimada intercepta y (Shao et al., 2016).

$$
\mathrm{SpO}_{2}=\alpha \times \mathrm{R}+\beta
$$

Para executar o procedimento de regressão linear, o software é iniciado e a resposta para a pergunta "É calibração? (s/n/r)" é a opção "r". Esse procedimento executa a função calcula_regressao() presente no software proposto, que foi desenvolvida para automatizar o cálculo da regressão linear. Antes de executar a função o software lê os arquivos RR_XXXX_1.txt e SpO2_XXXX_1.txt, RR_XXXX_2.txt e SpO2_XXXX_2.txt para gerar os 24 pares de dados referentes aos valores da taxa $\mathrm{R}$ e da leituras $\mathrm{SpO}_{2}$ do oxímetro de referência. Então, os 24 pares de dados são enviados para a função calcula_regressao(), que retorna como saída os valores de $\alpha$ e $\beta$. Tais valores são armazenados em dois arquivos distintos alfa.txt e beta.txt, para posteriormente serem aplicados na Eq. (1). Com os coeficientes $\alpha$ e $\beta$ calculados o processo de calibração é finalizado.

\subsubsection{Função para o cálculo da regressão linear}

A Fig. 4 apresenta o código fonte utilizado na solução proposta para o cálculo da regressão linear.

A função calcula_regressao() foi adaptada de Sharma (2015), que recebe dois arrays (eixoX e eixoY) que são construídos com os valores armazenados nos arquivos criados durante o processo de calibração.

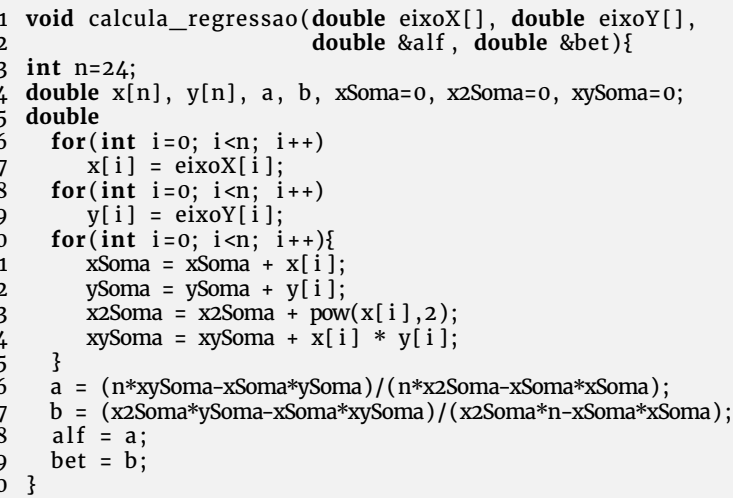

Figura 4: Código-fonte da função que calcula a regressão linear.

$\mathrm{O}$ eixoX corresponde a taxa $\mathrm{R}$ e o eixoY corresponde ao valor da $\mathrm{SpO}_{2}$. Como saída a função altera os valores de $\alpha$ e $\beta$, que estão armazenados nos arquivos alfa.txt e beta.txt e posteriormente são aplicados na Eq. (1) para o cálculo da $\mathrm{SpO}_{2}$.

Para validar a função calcula_regressao() foram utilizados os nove conjuntos de vinte e quatro pares de dados (taxa $\mathrm{R}$ e SpO2, respectivamente) obtidos nos processos de calibração. Para cada conjunto de dados foram calculados os valores de $\alpha$ e $\beta$ utilizando a função da solução proposta. Os mesmos nove conjuntos de dados foram adicionados, individualmente, no software GraphPad Software (2017) e a regressão linear foi calculada. Em todos os nove conjuntos de dados os valores de $\alpha$ e $\beta$ obtidos pela função calcula_regressao() e pelo Graph Pad foram iguais.

\subsection{Software proposto}

O software da solução proposta foi desenvolvido em $\mathrm{C}++$, por ser uma linguagem multiparadigma, flexível e multiplataforma. Foram utilizados apenas softwares livres como Raspbian, OpenCV, FFTW, RaspicamCV, entre outros. A Fig. 5, mostra o fluxo de processamento do software, desde a captura da imagem do indivíduo monitorado até a obtenção da taxa de saturação de oxigênio e frequência cardíaca. Como o desenvolvimento do software faz parte de um projeto de mestrado, o custo do desenvolvimento não foi computado. Fluxo do software proposto foi dividido em três partes para facilitar o entendimento. As etapas A a E constituem a primeira parte do processamento. As etapas F, G e $\mathrm{H}$ constituem a segunda parte do processamento que realiza a mensuração da frequência cardíaca e as etapas I e J constituem a terceira parte do processamento que mensuração da $\mathrm{SpO}_{2}$.

\subsubsection{Parte I - Etapas de A a E}

As etapas de $\mathrm{A}$ a $\mathrm{E}$ correspondem a 10 segundos de processamento, isto significa que elas são executadas 1 vez para cada um dos 300 frames. A Fig. 6 resume as etapas da parte I. 


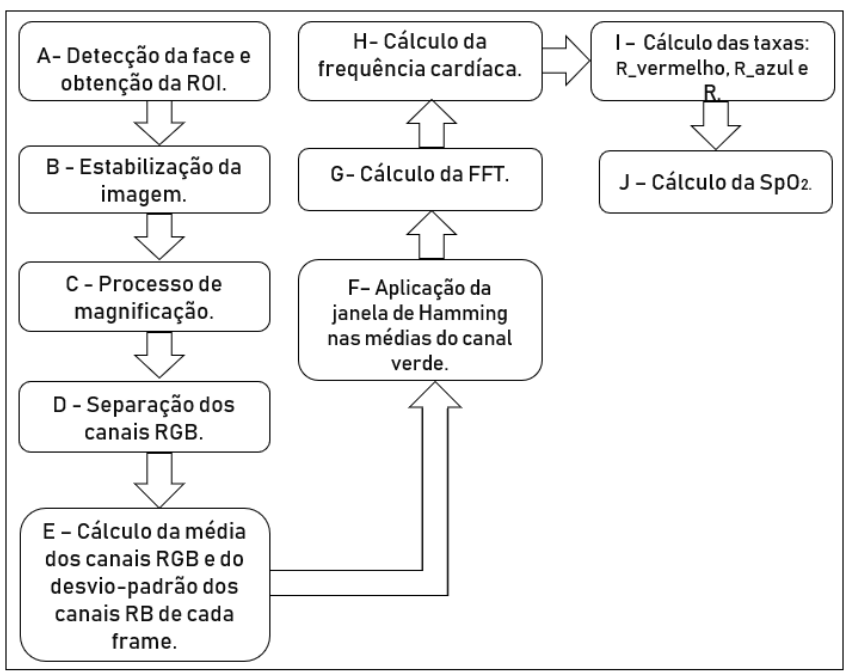

Figura 5: Esquema de fluxo do processamento do sinal fotopletismográfico realizado pela solução proposta.

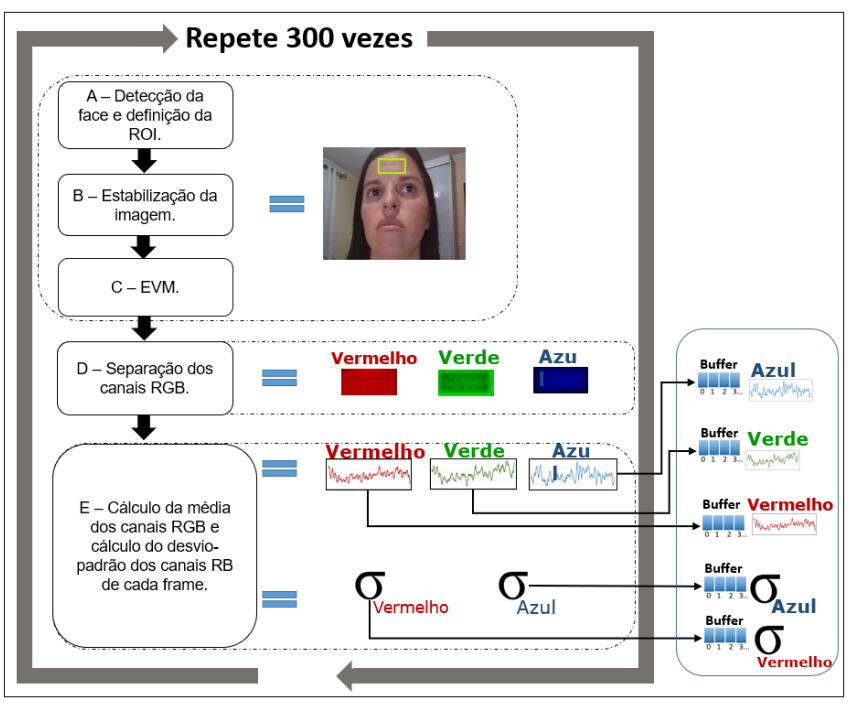

Figura 6: Processamento - Parte I - Etapas de A a E do software proposto.

3.5.1.1 Etapa (A): detecção da face e definição da ROI. Ao iniciar o software, a primeira etapa executada é a detecção da face e a definição da região de interesse (ROI). Para isso, é utilizada a classe pré-treinada chamada CascadeClassifier da biblioteca de visão computacional OpenCV.

A testa foi escolhida como ROI por ser uma região altamente vascularizada e também pelo fato de ocorrer menos reposicionamento do ROI com a testa em comparação com outras regiões, como olhos e boca (Simioni, 2015). A Fig. 7 é um exemplo de detecção da face e definição da ROI feita pela solução proposta.

3.5.1.2 Etapa (B): estabilização de imagem. Segundo Simioni (2015), o algoritmo de estabilização de imagem,

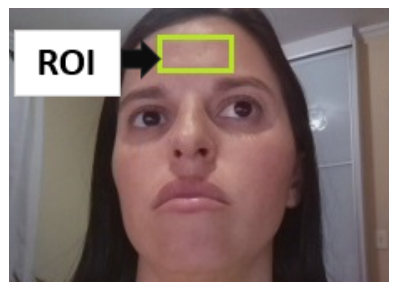

Figura 7: Detecção da face e seleção da ROI.

proposto por ele em sua dissertação, é parte fundamental para o sistema de monitoramento de frequência cardíaca. O objetivo do algoritmo é reduzir os ruídos gerados na imagem devido ao reposicionamento da ROI e diminuir a quantidade de imagens processadas pela solução.

Após a detecção da ROI, a imagem é submetida ao processo de estabilização, em que é comparada com a imagem anteriormente capturada. Se pelo menos $95 \%$ da quantidade da intensidade de cor da nova imagem for similar à quantidade da imagem anterior, a imagem nova é descartada. Simioni (2015) afirma que esse processo reduz em $40 \%$ a utilização do processador, se comparado com o processamento de todos os frames.

3.5.1.3 Etapa (C): processo de magnificação. Após a estabilização da imagem, a ROI é enviada para o algoritmo EVM. Ao receber a ROI, a operação resize down é realizada, a qual reduz o tamanho da imagem, e a operação blur é usada como filtro espacial para reduzir as altas frequências. Então, são aplicados dois filtros passa baixa IIR para limitar a amplificação das frequências, onde as frequências abaixo de 45 e acima de 180 batimentos por minuto são setadas como zero, e o frame é refeito utilizando a inversa da transformada de Fourier.

Após ser filtrada a imagem passa pelo processo de amplificação, onde o resultado do filtro temporal é multiplicado por um fator $\alpha$, o que resulta na magnificação da variação de cor. Por fim, a imagem é submetida a operação resize up retornando ao tamanho original e sendo adicionada novamente ao frame.

3.5.1.4 Etapa (D): separação do canais RGB. Finalizado o processamento do frame pelo algoritmo de EVM, os canais RGB (vermelho, verde e azul) são separados usando a função split da biblioteca OpenCV.

3.5.1.5 Etapa (E): cálculo das médias e dos desvios-padrão dos frames. Para cada canal (RGB), separadamente, foi calculada a média de intensidade de cor dos pixels de cada frame. O resultado foi armazenado em um array para cada uma das três cores RGB. Para isso foi utilizada a função mean da biblioteca OpenCV. Essas médias correspondem à intensidade de cor de cada canal no frame.

Para obter a $\mathrm{SpO}_{2}$ é necessário calcular o desviopadrão das intensidades de cor de cada frame dos canais vermelho e azul. Para isso, foi utilizada a função meanStdDev da biblioteca OpenCV. O resultado foi armazenado em um array para o canal da cor vermelha e outro para o canal da cor azul. Esses arrays são uti- 
lizados na etapa I para calcular os componentes AC e DC.

\subsubsection{Parte II - Etapas de F, G e H - Mensuração da frequência cardíaca}

Para obter a frequência cardíaca apenas as médias de intensidades de cor do canal verde são utilizadas. A Fig. 8 ilustra o esquema para a obtenção da frequência cardíaca ao executar as etapas F e G do software proposto.

3.5.2.1 Etapa (F): aplicação da janela de Hamming. Nessa etapa o array com as médias do canal verde, armazenadas na etapa anterior é divido em três partes, sendo uma com overlap de $50 \%$ das anteriores. Cada parte é filtrada aplicando a janela de Hamming. Segundo Simioni (2015) para evitar a inserção de ruídos de alta frequência, é aplicada a janela de Hamming ao array que armazena o sinal obtido do canal verde. Cada valor do vetor é multiplicado pelo resultado da Eq. (2).

$$
w[n]=0.54-0.46 * \cos \left(\frac{2 * \pi * n}{N-1}\right)
$$

Onde w é o array que armazena o sinal do canal verde após a aplicação da janela de Hamming, $N$ é o número de amostras e $n$ é a posição no array com as médias originais.
3.5.2.2 Etapa (G): cálculo da FFT. Após aplicada a janela de Hamming ao sinal do canal verde, é utilizada a biblioteca FFTW3 para calcular a transformada de Fourier. Logo após, é calculado o espectro de potência da FFT. Para finalizar o processo de transformação do domínio do tempo para o domínio da frequência, é calcula a média dos três espectros. Assim, os ruídos são reduzidos e os sinal de interesse é evidenciado.

No espectro de potência, é aplicado um filtro ideal que delimita a faixa de frequência diretamente na FFT, onde define-se o índice mínimo (idx_fft_mínimo) e índice máximo (idx_fft_máximo) da FFT.

A Eq. (3) determina a resolução "r" que corresponde ao valor da taxa de captura em frames por segundo (FPS), multiplicado por sessenta, dividido pelo número de amostras $(\mathrm{N})$.

As Eqs. (4) e (5) descrevem como são calculados esses índices, sendo bpm_mínimo a menor frequência cardíaca possível, bpm_máximo a maior frequência cardíaca possível ambos divididos pelo valor da resolução (r) obtido na Eq. (3).

$$
\begin{gathered}
r=\frac{60 * F P S}{N} \\
i d x \_f f t \text { minimo }=\frac{b p m \_ \text {minimo }}{r}
\end{gathered}
$$

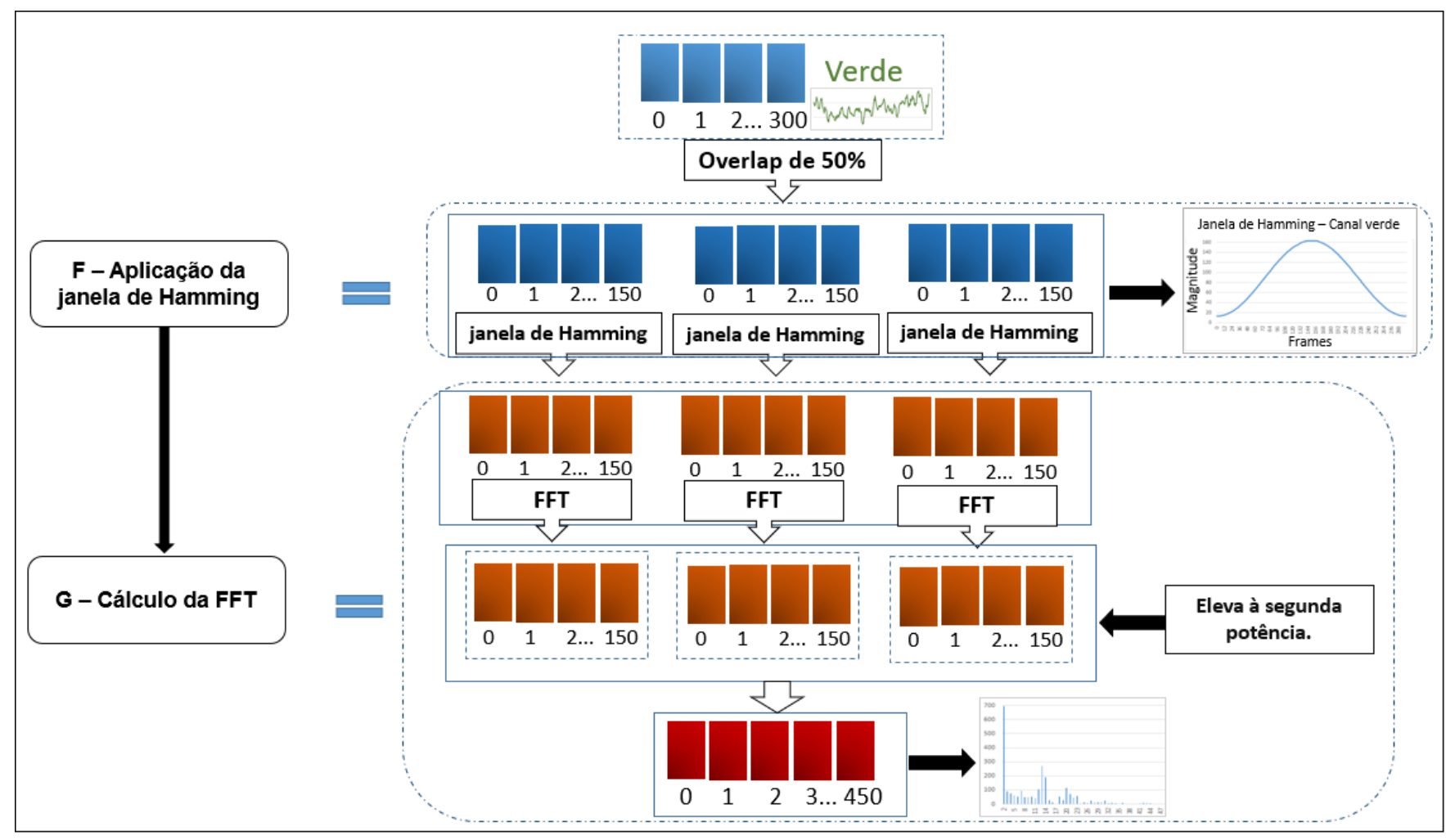

Figura 8: Processamento - Parte II - Etapas F e G - Obtenção da frequência cardíaca. 


$$
i d x \_f f t \_ \text {maximo }=\frac{b p m \_ \text {maximo }}{r}
$$

3.5.2.3 Etapa (H): cálculo da frequência cardíaca. Com os índices mínimo e máximo definidos, é realizada a detecção de maior pico dentro dos limites calculados. Localizado o índice da FFT correspondente ao maior pico é estimada a frequência cardíaca com a Eq. (6), onde $\mathrm{N}$ é o número de amostras, FPS é a taxa de captura e idx_fft é a posição na FFT onde está o maior pico do intervalo. A Fig. 9 ilustra o instante de tempo 234 segundos do experimento para validar a FC onde a leitura da FC do software proposto é igual a leitura apresentada pelo oxímetro de pulso de referência.

$$
b p m=\frac{F P S * 60 * i d x \_f f t}{N}
$$

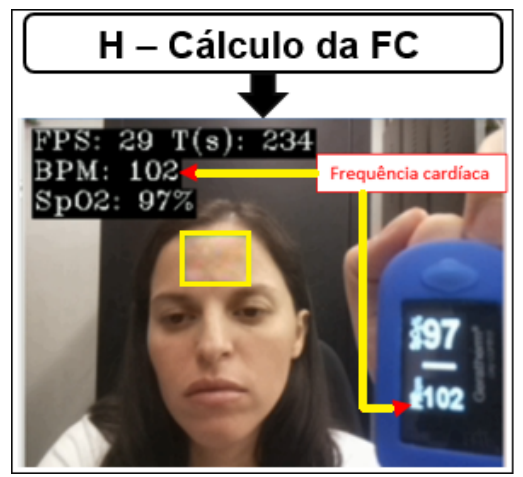

Figura 9: Exemplo de obtenção da frequência cardíaca.

\subsubsection{Parte III - Etapas de I e J - Mensuração da $\mathrm{SpO}_{2}$}

A Fig. 10 apresenta as etapas I e J para a obtenção da taxa de saturação de oxigênio da solução proposta.

3.5.3.1 Etapa (I): cálculo das taxas $R \_v e r m e l h o, ~ R \_a z u l e ~ R$. A taxa $\mathbf{R}$ é a relação normalizada da intensidade de luz vermelha e infravermelha transmitida/absorvida nos oxímetros de pulso convencionais (Shao et al., 2016), (Lin et al., 2016) e (Rusch et al., 1996).

Conforme afirmam Scully et al. (2012), Tarassenko et al. (2014) e Bal (2015), o canal azul equivale à luz infravermelha, e o canal vermelho representa a luz vermelha empregadas nos oxímetros convencionais. Portanto, para essa pesquisa, foram utilizados os canais vermelho e azul que compõem cada frame em substituição as luzes vermelha e infravermelha utilizadas no oxímetro de pulso com contato.

Para obter a taxa $\mathbf{R}$ primeiro é necessário estimar a taxa de absorção dos canais vermelho (R_vermelho) e azul (R_azul) (Bal, 2015). Segundo Scully et al. (2012), o componente AC é obtido por meio da definição do desvio-padrão da intensidade de cor dos canais vermelho e azul, para cada frame. Em uma janela de tempo de 10 segundos, com uma taxa de captura de 30 FPS,

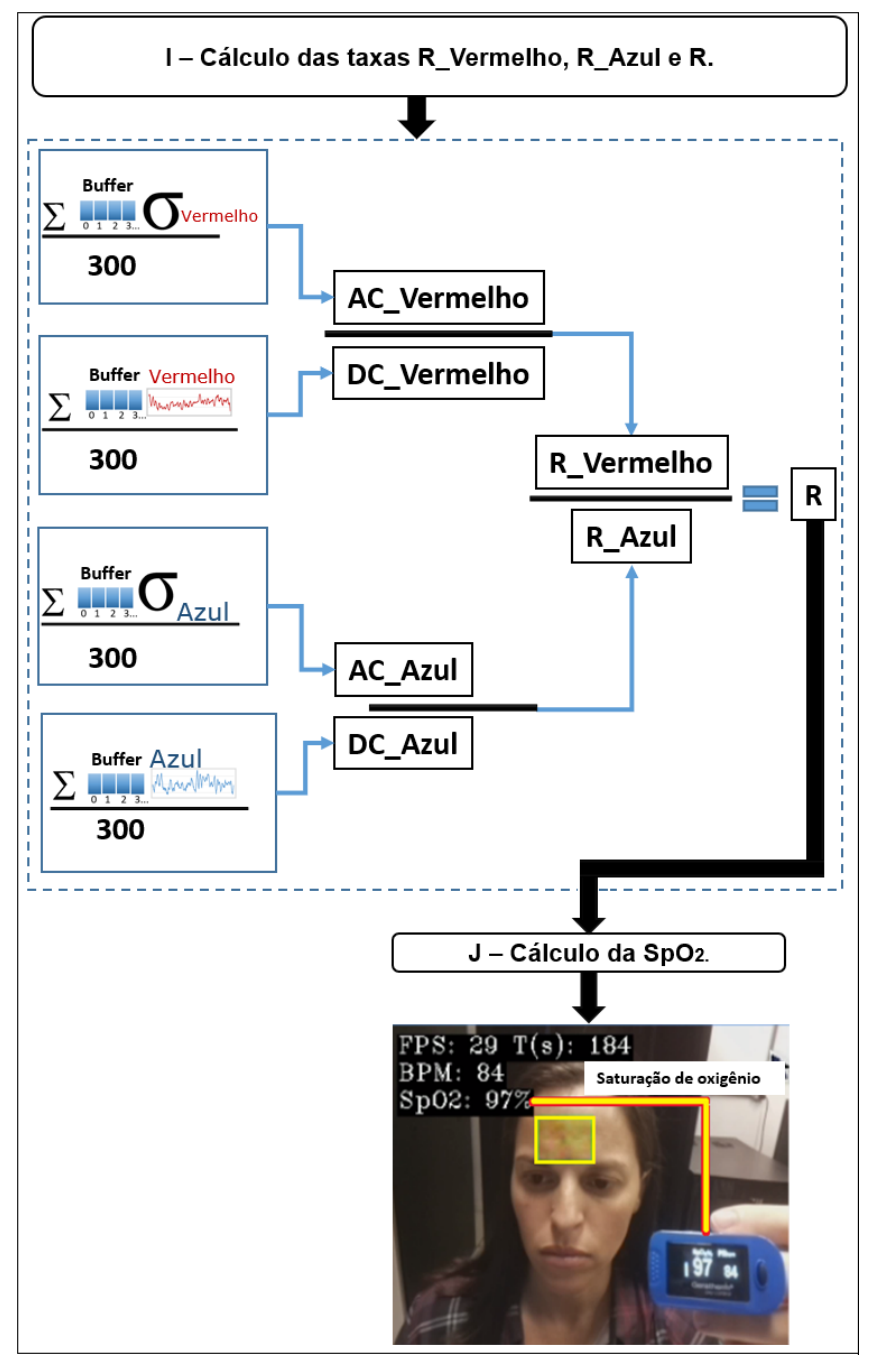

Figura 10: Processamento - Parte III - Etapas de I e J obtenção da $\mathrm{SpO}_{2}$.

foram obtidos 300 frames.

Com a função meanStdDev, da biblioteca OpenCV, foi calculada a média de intensidade de cor das cores vermelha e azul, que compõem cada um dos 300 frames. Os valores das médias foram armazenadas em um array de 300 posições para cada cor. Para cada array foi obtida a média dos valores das 300 posições, resultando no componente DC_vermelho e DC_azul. A função meanStdDev também retorna o desvio-padrão da intensidade de cor de cada frame, o qual corresponde ao componente AC. Os valores dos desvios-padrão foram armazenados em um array de 300 posições para cada cor. Para cada um dos arrays foi obtida a média dos valores das 300 posições, resultando nos componentes AC_vermelho e AC_azul. As taxas R_vermelho e R_azul são obtidas pelas Eqs. (7) e (8). Sendo a taxa $\mathbf{R}$ obtida pela Eq. (9).

$$
R_{\text {vermelho }}=\frac{A C_{\text {vermelho }}}{D C_{\text {vermelho }}}
$$




$$
\begin{aligned}
& R_{\mathrm{azul}}=\frac{A C_{\mathrm{azul}}}{D C_{\mathrm{azul}}} \\
& R=\frac{R_{\mathrm{vermelho}}}{R_{\text {azul }}}
\end{aligned}
$$

3.5.3.2 Etapa (J): cálculo da taxa de saturação de oxigênio. $\mathrm{O}$ valor da $\mathrm{SpO}_{2}$ é obtido através da Eq. (10).

$$
\mathrm{SpO}_{2}=\alpha * \mathrm{R}+\beta
$$

Nesta pesquisa foi utilizada a equação linear de melhor ajuste para obter os coeficientes $\alpha$ e $\beta$, similarmente aos estudos de Scully et al. (2012), Kong et al. (2013), Tarassenko et al. (2014), Bal (2015), Addison (2016), Shao et al. (2016) e Fan and Li (2018). Como já mencionado foi realizada a calibração para dada voluntário para obter os coeficientes $\alpha \mathrm{e} \beta$.

\section{Análise dos dados}

Para verificar a exatidão da solução proposta os valores da $\mathrm{SpO}_{2}$ e da $\mathrm{FC}$ apresentados pelo software proposto foram comparados com os valores obtidos por um oxímetro de pulso de referência. A Tabela 2 é um exemplo de como os dados de um experimento foram registrados. Onde $\mathrm{SpO}_{2}$ ens e FC_ens correspondem aos valores da $\mathrm{SpO}_{2}$ e FC obtidos pela solução proposta, respectivamente e $\mathbf{S p O}_{2}$ ref e $\mathbf{F C}$ _ref correspondem aos valores da $\mathrm{SpO}_{2}$ e FC obtidos pelo oxímetro de pulso de referência, respectivamente. O tempo foi registrado em segundos.

\begin{tabular}{|c|c|c|c|c|}
\hline Tempo & $\mathrm{SpO}_{2} \_$ens & $\mathrm{SpO}_{2} \_$ref & FC_ens & FC_ref \\
\hline 10 & 95 & 97 & 80 & 73 \\
\hline 20 & 95 & 96 & 46 & 70 \\
\hline 30 & 95 & 96 & 65 & 70 \\
\hline 40 & 95 & 95 & 77 & 69 \\
\hline 50 & 96 & 95 & 47 & 69 \\
\hline - & . & . & . & - \\
\hline - & - & - & . & . \\
\hline . & . & . & . & • \\
\hline 230 & 96 & 96 & 47 & 71 \\
\hline 240 & 96 & 96 & 47 & 72 \\
\hline 250 & 95 & 97 & 47 & 70 \\
\hline 260 & 95 & 96 & 89 & 76 \\
\hline 270 & 96 & 97 & 47 & 70 \\
\hline 280 & 96 & 97 & 53 & 71 \\
\hline 290 & 95 & 96 & 83 & 74 \\
\hline 300 & 95 & 96 & 71 & 69 \\
\hline
\end{tabular}

Tabela 2: Exemplo de planilha com os dados de um experimento.

\subsection{Análise dos dados da $\mathrm{SpO}_{2}$}

Para registrar os valores obtidos pela solução proposta e pelo oxímetro, todos os experimentos foram grava- dos em vídeo. Cada um dos vídeos foi analisado e os valores da $\mathrm{SpO}_{2}$ foram registrados em uma planilha, conforme Tabela 2 . A cada 10 segundos a solução proposta atualizava os valores da $\mathrm{SpO}_{2}$ e na tela de saída da solução proposta era exibido o tempo em segundo. A cada 10 segundos os valores de ambos os métodos foram registrados na planilha. Cada experimento gerou uma planilha com 30 pares de dados correspondentes as medições da $\mathrm{SpO}_{2}$ para cada participante.

\subsection{Métrica de avaliação da $\mathrm{SpO}_{2}$}

A norma NBR ISO 80601-2-61:2015 ABNT (2015) afirma que a exatidão da $\mathrm{SpO}_{2}$ dos equipamentos para oximetria de pulso o ( $\mathrm{A}_{\mathrm{rms}}$ ) deve ser calculado em termos da diferença em valor quadrático médio (vqm) entre os valores medidos $\left(\mathrm{SpO}_{2 \mathrm{i}}\right)$ e o valor de referência $\left(\mathrm{S}_{\mathrm{Ri}}\right)$, conforme Eq. (11). Onde $A_{r m s}$ é a exatidão, $n$ é o número de pares dados da amostra contidos na faixa de interesse, $\mathrm{SpO}_{2 \mathrm{i}}$ é o i-ésimo valor da saturação de oxigênio dado pelo equipamento ensaiado, $\mathrm{S}_{\mathrm{Ri}}$ é o i-ésimo valor da saturação de oxigênio dado pelo equipamento de referência.

$$
A_{r m s}=\sqrt{\frac{\sum_{i=1}^{n}\left(S p O_{2 i}-S_{R i}\right)^{2}}{n}}
$$

Portanto, para cada linha das tabelas com dos dados da $\mathrm{SpO}_{2}$ foi obtida a diferença entre $\mathrm{SpO}_{2}$ ens e $\mathrm{SpO}_{2}$ ref. O resultado dessa operação foi elevado a segunda potência para obter o valor do quadrado da diferença. Com os quadrados das diferenças determinados foi calculada a média dos quadrados. Então, foi calculada a raiz quadrada da média dos quadrados das diferenças para obter o Arms, conforme Eq. (11).

Esse procedimento foi feito com os dados obtidos em cada um dos experimentos e seus valores são apresentados na Seção de Resultados.

Para cada tipo de experimento (E1 ou E2) foi obtido o Arms geral. Para isso, foram somados os quadrados das diferenças dos experimentos (separados por experimento) de todos os voluntários e em seguida calculado o vqm. Então, o valor do vqm foi aplicado na Eq. (11) resultando no Arms geral para cada experimento.

\subsection{Análise dos dados da frequência cardíaca}

Para validar a obtenção da frequência cardíaca pela solução proposta, utilizando as mesmas métricas descritas por Simioni (2015), foram utilizados os dados de um vídeo de cinco minutos de um indivíduo de pele branca, onde a frequência cardíaca foi simultaneamente monitorada pela solução proposta e por um oxímetro de pulso comercial. Desse vídeo foram extraídas 200 amostras da FC. Os valores da FC obtidos por ambos os métodos foram registrado em uma planilha, onde FC_ens são os valores obtidos pela solução proposta e FC_ref são os valores da FC obtidos pelo oxímetro de pulso de referência. Os valores foram comparados entre 
si e calculada a porcentagem em que os valores obtidos pela solução proposta estavam dentro da margem de resolução \pm 6 BMP.

Visando melhorar a avaliação da solução proposta, além da avaliação similar a proposta por Simioni (2015), foi realizada uma análise das aferições da FC dos 9 participantes da pesquisa. Conforme realizado para a aferição da $\mathrm{SpO}_{2}$, cada experimento gerou uma planilha com 30 pares de dados correspondentes as medições da FC para cada participante. Para a análise da FC foram utilizados os dados do primeiro experimento e a técnica de concordância de Altman and Bland (1983) foi empregada. O método de Bland e Altman é uma técnica estatística para comparação de dois métodos, usada para mensuração de variáveis clínicas. Para aplicar a técnica são calculas as diferenças entre as medidas obtidas pelos dois métodos e a média e o desvio padrão dessas diferenças. Se os valores das diferenças têm distribuição normal, é esperado que $95 \%$ dos valores da diferença fiquem entre a média \pm 2 SD. Esse intervalo é chamado de "limite de concordância". Se a faixa de variação desse intervalo for grande, conclui-se que não há concordância entre os dois métodos (Minicucci et al., 2007).

\section{Resultados}

Nessa seção são apresentados os resultados obtidos com os experimentos.

\subsection{Resultados para a $\mathrm{SpO}_{2}$}

Como já mencionado a norma NBR ISO 80601-261:2015 ABNT (2015) afirma que a exatidão da $\mathrm{SpO}_{2}$ do equipamento ( $\mathrm{A}_{\mathrm{rms}}$ ) deve ser calculada em termos da diferença em valor quadrático médio (vqm) entre os valores medidos pelo equipamento ensaiado e o valor de referência medidos pelo equipamento de referência.

A norma NBR ISO 80601-2-61:2015 ABNT (2015) também afirma que para validar um equipamento para monitoramento de $\mathrm{SpO}_{2}$ o Arms deve ser menor que $2 \%$ da faixa declarada.

O experimento 1 corresponde ao procedimento de monitoramento de cinco minutos, onde o indivíduo respira normalmente durante todo o decorrer do tempo de experimento. $O$ Arms geral do experimento 1 foi de $0,99 \%$ sobre a faixa de saturação de 99 e $92 \%$, considerando os dados de 09 voluntários. A Tabela 3 apresenta o Arms obtido para cada voluntário para o experimento 1.

O experimento 2 corresponde ao procedimento de monitoramento de cinco minutos, onde após decorridos os dez segundos iniciais do experimento foi solicitado ao indivíduo que segurasse a respiração até se sentir desconfortável para que ocorresse a diminuição do nível de oxigênio no sangue. Passado o período que o voluntário ficou sem respirar foi solicitado que o mesmo respirasse normalmente, durante o restante de tempo de gravação do vídeo, para que a taxa de saturação voltasse aos valores normais. O Arms geral do experimento 2 foi de $1,09 \%$ sobre a faixa de saturação de 99 e $92 \%$,
Tabela 3: Valor do Arms para cada voluntário Exp. 1.

\begin{tabular}{|c|c|c|c|c|}
\hline ID & Sexo & $\begin{array}{c}\text { Quadrado } \\
\text { das } \\
\text { diferenças }\end{array}$ & $\begin{array}{c}\text { Média } \\
\text { das } \\
\text { diferenças }\end{array}$ & Arms\% \\
\hline 1 & F & 2 & 0,066 & 0,258199 \\
\hline 2 & M & 60 & 2 & 1,414213 \\
\hline 3 & M & 0 & 0 & 0 \\
\hline 4 & M & 23 & 0,766 & 0,875595 \\
\hline 5 & F & 5 & 0,166 & 0,408248 \\
\hline 6 & F & 29 & 0,96 & 0,983192 \\
\hline 7 & M & 41 & 1,366 & 1,169045 \\
\hline 8 & F & 27 & 0,9 & 0,948683 \\
\hline 9 & F & 80 & 2,66 & 1,632993 \\
\hline
\end{tabular}

considerando os dados de 08 voluntários.

$O$ voluntário de ID 07 não conseguiu executar esse experimento, ele não conseguia ficar sem respirar por mais de 10 segundos, portanto seus dados para esse experimento foram desconsiderados. A Tabela 4 apresenta o Arms obtido para cada voluntário para o experimento 2 .

Tabela 4: Valor do Arms para cada voluntário Exp. 2.

\begin{tabular}{|c|c|c|c|c|}
\hline ID & Sexo & $\begin{array}{c}\text { Quadrado } \\
\text { das } \\
\text { diferenças }\end{array}$ & $\begin{array}{c}\text { Média } \\
\text { das } \\
\text { diferenças }\end{array}$ & Arms\% \\
\hline 1 & F & 25 & 0,83 & 0,912871 \\
\hline 2 & M & 29 & 0,96 & 0,983192 \\
\hline 3 & M & 0 & 0 & 0 \\
\hline 4 & M & 23 & 0,766 & 0,875595 \\
\hline 5 & F & 7 & 0,233 & 0,483045 \\
\hline 6 & F & 64 & 2,133 & 1,460593 \\
\hline 8 & F & 21 & 0,7 & 0,836660 \\
\hline 9 & F & 118 & 3,933 & 1,983263 \\
\hline
\end{tabular}

O voluntário ID 3 apresentou valores constantes para a $\mathrm{SpO}_{2}$ tanto nas leituras da solução proposta quanto nas leituras do oxímetro de pulso de referência, nos dois experimentos. Não foi possível determinar o motivo da não variação nos níveis de oxigênio no sangue desse indivíduo, mesmo o voluntário ficando em apneia por alguns segundos. Portanto, as leituras da $\mathrm{SpO}_{2}$ desse voluntário foram retiradas do cálculo do Arms geral para verificar qual seria a interferência no resultado final. Ficando os resultados da seguinte forma: para o experimento 1 o Arms geral passou de $0,99 \%$ para $1,11 \%$ sobre a faixa de saturação de 99 a $92 \%$. Para o experimento 2 o Arms passou de $1,09 \%$ para $1,16 \%$ sobre a faixa de saturação de 99 a $92 \%$. Em ambos os resultados houve uma pequena variação, mas a solução proposta ainda ficou com $A_{\text {rms }}$ abaixo dos $2 \%$ exigidos pela norma.

Ao aplicar o método de Bland-Altman para verificar a concordância entre os dois métodos em relação a aferição da $\mathrm{SpO}_{2}$, foi obtido o gráfico apresentado na Fig. 11. Nesse gráfico é possível verificar que as diferenças estão dentro do limite de concordância isso indica um bom grau de concordância entre a solução proposta e o oxímetro de pulso com contato físico. 


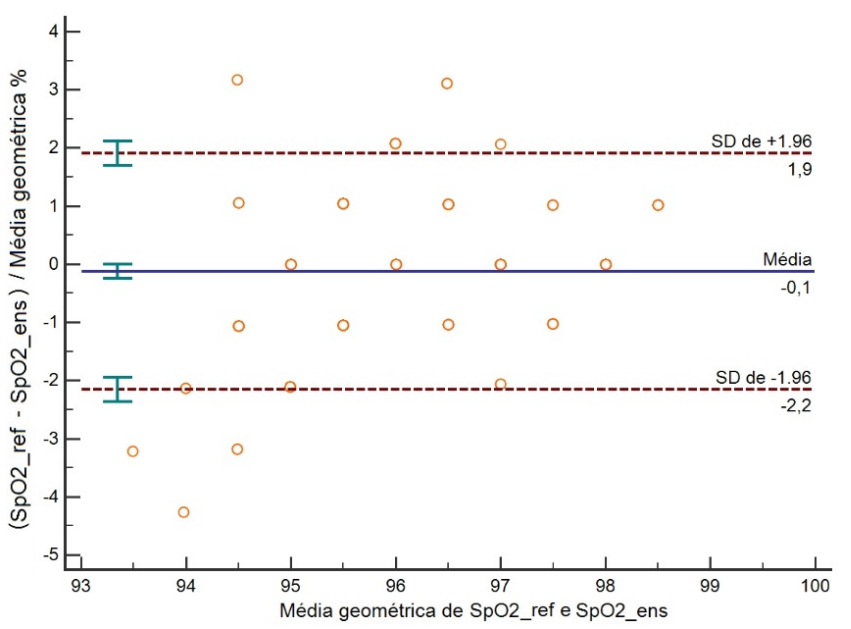

Figura 11: Gráfico de Bland-Altman com dados da $\mathrm{SpO}_{2}$ dos nove participantes obtidos no experimento 1 .

\subsection{Resultados para FC}

Das 200 amostras registrada no experimento para a avaliação da FC 195 encontraram-se dentro da faixa de resolução de \pm 6 BMP obtida pela Eq. (3). Sendo assim a taxa de acerto da obtenção da FC pela solução proposta é de $97,5 \%$, similar a taxa apresentada por Simioni (2015).

Para verificar a concordância das leituras apresentadas pela solução proposta e o oxímetro de pulso de referência. Os dados do experimento 1 dos nove participantes foram plotados no gráfico de Bland-Altman, conforme Fig. 12.

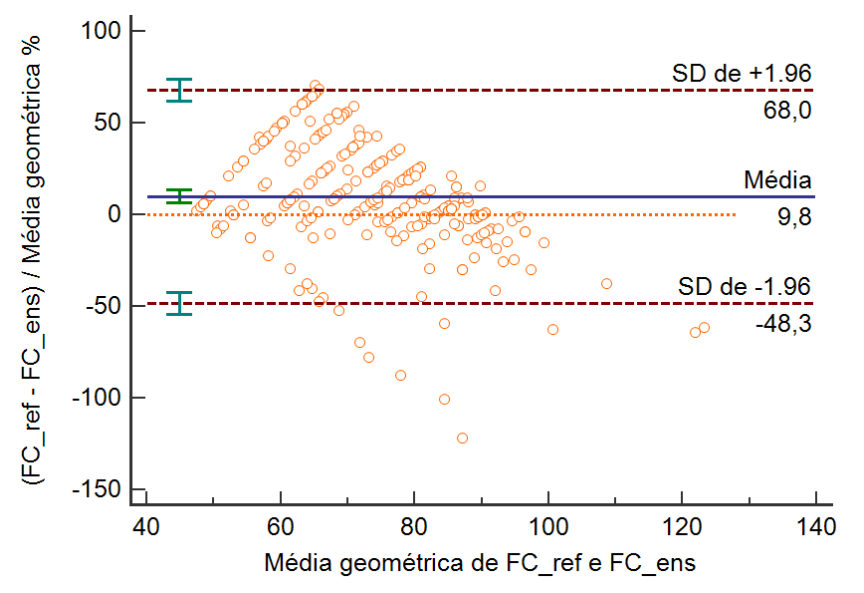

Figura 12: Gráfico de Bland-Altman com dados da FC dos nove participantes obtidos no experimento 1 .

É possível verificar na Fig. 12 que a faixa de variação do limite de concordância entre os dois métodos é muito grande, isso demonstra que a solução proposta não apresenta um bom grau de concordância ao se comparar com o oxímetro de pulso com contato físico.

\section{Conclusão}

Vários estudos versam sobre a obtenção da taxa de saturação de oxigênio e frequência cardíaca utilizando imagens de vídeo, porém necessitam de equipamentos como câmeras e fonte de luz específicas, computadores com alto desempenho e software proprietário. Essas características tornam o custo da solução elevado. Do total de estudos levantados, na revisão da literatura realizado para esse artigo, apenas um artigo informa a mensuração de $\mathrm{SpO}_{2}$ e a $\mathrm{FC}$ em tempo real, porém utilizando fonte de luz específica e sem menção ao hardware utilizado para obter e processar as imagens, dificultando a comparação com a proposta desse artigo. $97,5 \%$ das leituras da frequência cardíaca feitas pela solução proposta ficaram dentro de faixa de resolução \pm 6 BMP ao se comparar com as leituras do oxímetro de referência, conforme as métricas descritas por Simioni (2015). Entretanto, ao verificar a concordância entre a solução proposta e o oxímetro de referência através do gráfico de Bland-Altman, o grau de concordância obtido não foi satisfatório. Sendo assim, para a aferição da FC a solução proposta deve ser melhorada. Foi possível mensurar a acurácia da solução proposta comparando os valores obtidos da $\mathrm{SpO}_{2}$ como as leituras apresentadas por um oxímetro de pulso como referência. Sendo que a acurácia sobre a faixa de saturação de 99 a $92 \%$ foi de $0,99 \%$ e com o processo de apneia a acurácia ficou em $1,09 \%$, valor abaixo dos $2 \%$ exigido pela norma. Mas para cada indivíduo monitorado foi necessário realizar o processo de calibração prévio. Esse é um problema atual que ainda não foi totalmente solucionado e necessita de mais estudos. Portanto, a solução proposta tem potencial para ser uma alternativa sem contato físico para o monitoramento da $\mathrm{SpO}_{2}$ e da FC.

\section{Referências}

ABNT (2015). NBR ISO 80601-2-61: Equipamentos eletromédico Parte 2-61: Requisitos particulares para a segurança básica e o desempenho essencial de equipamentos para oximetria de pulso, Technical report, Associação Brasileira de Normas Técnicas. Disponível em: https://www.abntcatalogo.com.br/norma. aspx? $\mathrm{ID}=332236$.

Addison, P. S. (2016). Modular continuous wavelet processing of biosignals: extracting heart rate and oxygen saturation from a video signal, Healthcare Technology Letters 3(2): 111-115. https://doi.org/10. 1049/htl.2015.0052.

Addison, P. S., Jacquel, D., Foo, D. M. H., Antunes, A. and Borg, U. R. (2017). Video-Based Physiologic Monitoring during an Acute Hypoxic Challenge: Heart Rate, Respiratory Rate, and Oxygen Saturation, Anesthesia and Analgesia 125(3): 860-873. https: //doi.org/10.1213/ane.0000000000001989.

Altman, D. G. and Bland, J. M. (1983). Measurement in medicine: The analysis of method comparison 
studies, The Statistician 32(3): 307. https://doi.org/ $10.2307 / 2987937$.

Bal, U. (2015). Non-contact estimation of heart rate and oxygen saturation using ambient light, Biomedical Optics Express 6(1): 86. https://doi . org/10.1364/BOE. 6.000086 .

Cobos-Torres, J.-C. and Abderrahim, M. (2017). Simple measurement of pulse oximetry using a standard color camera, 2017 40th International Conference on Telecommunications and Signal Processing (TSP), IEEE, pp. 452-455. https://doi.org/10.1109/tsp. 2017. 8076026.

Edwards Life Sciences (2002). UNDERSTANDING CONTINUOUS MIXED VENOUS OXYGEN SATURATION ( $\mathrm{SVO}_{2}$ 2) SWAN-GANZ OXIMETRY UNDERSTANDING CONTINUOUS MIXED VENOUS OXYGEN. Disponível em: http://ht.edwards.com/resourcegallery/ products/swanganz/pdfs/svo2edbook.pdf.

Fan, Q. and Li, K. (2018). Non-contact remote estimation of cardiovascular parameters, Biomedical Signal Processing and Control 40: 192-203. http://dx.doi . org $/ 10.1016 / \mathrm{j}$. bspc. 2017.09.022.

Fitzpatrick, T. B. (1988). The validity and practicality of sun-reactive skin types i through VI, Archives of Dermatology 124(6): 869-871. https://doi .org/10. 1001/archderm.1988.01670060015008.

GraphPad Software (2017). Quickcalcs. Disponível em: https://www.graphpad.com/quickcalcs/linear1/ (Acessado 1 de Abril de 2017).

Guazzi, A. R., Villarroel, M., Jorge, J., Daly, J., Frise, M. C., Robbins, P. A. and Tarassenko, L. (2015). Noncontact measurement of oxygen saturation with an RGB camera., Biomedical optics express 6(9): 33203338. https://doi.org/10.1364/BOE.6.003320.

Guyton, A. C. and John, E. H. (2010). Tratado de Fisiologia Médica, 12 edn, Elsevier, chapter 1, p. 29.

Humphreys, K., Ward, T. and Markham, C. (2007). Noncontact simultaneous dual wavelength photoplethysmography: A further step toward noncontact pulse oximetry, Review of Scientific Instruments 78(4). https://doi.org/10.1063/1.2724789.

Imms, R., Hu, S., Azorin-Peris, V., Trico, M. and Summers, R. (2014). A high performance biometric signal and image processing method to reveal blood perfusion towards 3D oxygen saturation mapping, Proc.SPIE p. 89470X. https://doi.org/10.1117/ 12.2044318

Kong, L., Zhao, Y., Dong, L., Jian, Y., Jin, X., Li, B., Feng, Y., Liu, M., Liu, X. and Wu, H. (2013). Noncontact detection of oxygen saturation based on visible light imaging device using ambient light, Optics Express 21(15): 17464. https : //doi . org/10. 1364/OE. 21. 017464.
Kooij, K. M. V. and Naber, M. (2019). An opensource remote heart rate imaging method with practical apparatus and algorithms, Behavior Research Methods 51(5): 2106-2119. https://doi.org/10.3758/ s13428-019-01256-8.

Li, J., Dunmire, B., Beach, K. W. and Leotta, D. F. (2013). A reflectance model for non-contact mapping of venous oxygen saturation using a CCD camera, Optics Communications 308: 78-84. https://doi.org/ 10.1016/j.optcom.2013.06.041.

Lin, B.-S., Huang, C.-Y., Chen, C.-Y. and Lin, J.-H. (2016). Design of a finger base-type pulse oximeter, Review of Scientific Instruments 87(1): 013108. https: //doi.org/10.1063/1.4940237.

Minicucci, M. F., Azevedo, P. S., Duarte, D. R., Matsubara, B. B., Matsubara, L. S., Campana, Á. O., Paiva, S. A. R. and Zornoff, L. A. M. (2007). Comparação de diferentes métodos para medida do tamanho do infarto experimental crônico em ratos, $\mathrm{Ar}$ quivos Brasileiros de Cardiologia 89(2): 93-98. https: //doi.org/10.1590/S0066-782X2007001400004.

Mishra, D., Priyadarshini, N., Chakraborty, S. and Sarkar, M. (2017). Blood Oxygen Saturation Measurement Using Polarization-Dependent Optical Sectioning, IEEE Sensors Journal 17(12): 3900-3908. https://doi .org/10.1109/JSEN . 2017.2698520.

Moço, A. V., Stuijk, S. and de Haan, G. (2018). New insights into the origin of remote PPG signals in visible light and infrared, Scientific Reports 8(1). https : //doi. org/10.1038/s41598-018-26068-2.

Nitzan, M. and Taitelbaum, H. (2008). The Measurement of Oxygen Saturation in Arterial and Venous Blood, Instrumentation \& Measurement Magazine, IEEE pp. 9-15. https://doi.org/10.1109/mim. 2008. 4534373.

O'Driscoll, B. R., Howard, L. S. and and, A. G. D. (2008). BTS guideline for emergency oxygen use in adult patients, Thorax 63(Supplement 6): vi1-vi68. https : //doi.org/10.1136/thx.2008.102947.

Poh, M.-Z. and Poh, Y. C. (2017). Validation of a standalone smartphone application for measuring heart rate using imaging photoplethysmography, Telemedicine and e-Health 23(8): 678-683. https://doi .org/ 10.1089/tmj. 2016.0230.

Rusch, T., Sankar, R. and Scharf., J. (1996). Signal processing methods for pulse oximetry, Computers in Biology and Medicine 26(2): 143 - 159. https://doi. org/10.1016/0010-4825(95)00049-6.

Scully, C. G., Lee, J., Meyer, J., Gorbach, A. M., Granquist-Fraser, D., Mendelson, Y. and Chon, K. H. (2012). Physiological parameter monitoring from optical recordings with a mobile phone, IEEE Transactions on Biomedical Engineering 59(2): 303-306. https: //doi.org/10.1109/TBME.2011.2163157. 
Shao, D., Liu, C., Tsow, F., Yang, Y., Du, Z., Iriya, R., Yu, H. and Tao, N. (2016). Noncontact monitoring of blood oxygen saturation using camera and dual-wavelength imaging system, IEEE Transactions on Biomedical Engineering 63(6): 1091-1098. https://doi.org/10.1109/tbme.2015.2481896.

Sharma, M. (2015). C++ program to linear fit the data using least squares method. Disponível em: https: //www . bragitoff . com/2015/09/page/2/.

Simioni, M. C. (2015). Monitoramento da Frequência Cardíaca via Método de Magnificação de Vídeo Euleriana em Tempo Real, Master's thesis, Universidade Tecnológica Federal do Paraná. Disponível em: http: //repositorio.utfpr.edu.br/jspui/handle/1/1373.

Tamura, T. (2019). Current progress of photoplethysmography and SPO2 for health monitoring, Biomedical Engineering Letters 9(1): 21-36. https://doi.org/ 10.1007/s13534-019-00097-w.

Tarassenko, L., Villarroel, M., Guazzi, A., Jorge, J., Clifton, D. a. and Pugh, C. (2014). Non-contact videobased vital sign monitoring using ambient light and auto-regressive models., Physiological measurement 35(5): 807-31. https://doi.org/10.1088/0967-3334/ $35 / 5 / 807$.

Tsai, H. Y., Huang, K. C., Chang, H. C., Yeh, J. L. A. and Chang, C. H. (2014). A noncontact skin oxygensaturation imaging system for measuring human tissue oxygen saturation, IEEE Transactions on Instrumentation and Measurement 63(11): 2620-2631. https : //doi.org/10.1109/TIM.2014.2312512.

Van Gastel, M., Stuijk, S. and De Haan, G. (2016). New principle for measuring arterial blood oxygenation, enabling motion-robust remote monitoring, Scientific Reports 6. https://doi.org/10.1038/srep38609.

Verkruysse, W., Bartula, M., Bresch, E., Rocque, M., Meftah, M. and Kirenko, I. (2017). Calibration of Contactless Pulse Oximetry, Anesthesia \& Analgesia . https://doi.org/10.1213/ane.0000000000001381.

Verkruysse, W., Svaasand, L. O. and Nelson, J. S. (2008). Remote plethysmographic imaging using ambient light., Optics Express 16(26): 21434-21445. https:// doi.org/10.1364/OE.16.021434.

Wang, W., den Brinker, A. C. and de Haan, G. (2018). Full video pulse extraction, Biomedical Optics Express 9(8): 3898. https://doi.org/10.1364/boe.9.003898.

Wang, Y.-H., Hung, C.-J., Shen, C.-H. and Chen, S.-J. (2010). A new oxygen saturation images of iris tissue, Proceedings of IEEE Sensors pp. 1386-1389. https:// doi.org/10.1109/icsens.2010.5690526.

Wieringa, F. P., Mastik, F. and Van Der Steen, A. F. W. (2005). Contactless multiple wavelength photoplethysmographic imaging: A first step toward "SpO2 camera" technology, Annals of Biomedical Engineering 33(8): 1034-1041. https://doi.org/10.1007/ s10439-005-5763-2. 